\title{
BEACON: AN APPLICATION OF IOT
}

\author{
V.S.Roshni ${ }^{1}$, K.Brinda ${ }^{2}$, S.Ranjani ${ }^{3}$
}

B.Tech, Electronics and Communication Engineering, SRM Institute of Science and Technology, Kanchipuram, Tamilnadu ${ }^{1}$.

B.Tech, Electronics and Communication Engineering, SRM Institute of Science and Technology, Kanchipuram, Tamilnadu ${ }^{2}$.

Assistant Professor(Sr.G), Faculty of Telecommunication Engineering, SRM Institute of Science and Technology, Kanchipuram, Tamilnadu ${ }^{3}$.

\begin{abstract}
Beacon is viewed as an indoor tracking system. It is an excellent way to monitor objects or people in a specific area. This can be accomplished by attaching a tag to an asset present in a particular area. Then by scattering Bluetooth signal, RSSI (Received Signal Strength Indicator) measures the strength of the scattered signal thereby resulting in position acquisition. With IOT flourishing, many researchers expect more than 20 billion connected devices by 2020. Also with the introduction of $5 G$ (Fifth generation) a request for data can be achieved in less than 1 millisecond which is four hundred times faster than the time taken for our eyes to blink. With all these inventions coming into fray beacon enabled smart cities isn't a dream.
\end{abstract}

Keywords: Beacon, RSSI, Indoor, Asset, IOT

\section{INTRODUCTION}

Almost 36 million people are blind and moreover $65 \%$ of the blind people are more than fifty years of age. These people are incarcerated for their disability making them unable to travel around the world. Many technologies like white cane and guide dogs have been implemented to enable the blind to navigate, but due to some ambiguities in these technologies the blind feel abandoned from this world. So scientists and engineers came out with amenities for blind in association with IOT and that is Beacon technology which is based on Indoor Positioning System.

Indoor Positioning System is a system that is used to track valuable objects within a specified area using radio waves, magnetic waves, acoustic signals or sensory information collected by mobile devices. A position of an object can be found out by measuring the distance between the object and an anchor node(nodes with known position eg: WIFI access points). While in GPS a method called Trilateration is used which is a widespread technology to date in the positioning system. This is mainly used in outdoor positioning system with the help of satellites in revolution around the earth.

A Beacon is a small BLE(Bluetooth Low Energy)device that can be powered by coin cells ,batteries or external power supply.BLE or Bluetooth smart uses Bluetooth 4.0 technology which operates between $2400 \mathrm{MHz}$ and $2485 \mathrm{MHz}$. It has been divided into 40 different channels and each channel spaced with 2MHz.It is low-cost low-power consumption, low-complexity and low-bandwidth technology .It stands for its unfazed penetration capabilities in society. Depending on varying functionality it is used for advertising to notify its presence, scanning for detection of smart devices and connected as a master or a slave for efficient transmission of data. When a connection is required BLE Master connects to various BLE slaves in star piconet topology whereas a mediocre Bluetooth could connect only seven Bluetooth slaves in other topologies. Furthermore to emphasize its importance Apple Corporation provided Beacon supports in ios version 7.0 and Google provided in its 4.3 version 
but windows operating system doesn't support this technology, this paradigm may shift in near future because of its growing popularity. Beacon differs from other technologies as it is a one-way communication between a transmitter and a receiver. Here the receiver is an android mobile with an app downloaded in it. This ensures that only the installed app can monitor people potential against their will when walked past the transmitters. Power consumption in phones is a major factor in this technology as a report revealed that older mobile phones drew more energy from its battery when they were in Beacon network whereas new android mobiles made use of optimal source of energy. The major factors contributing to energy consumption is the number of beacons in a specific area and the mobile's energy consumption. These factors must be kept in mind for efficient use of this technology.

\section{COMPONENTS USED IMPLEMENTATION}

For implementing this above mentioned technology there is a requirement of certain components, they are: Onyx beacon(supports both Android and Apple iOS) or any other high performance beacons and mobile application installed in the customer's mobile.

\section{(B) ONYXBEACON}

\section{Beacon One}

Available since 2014

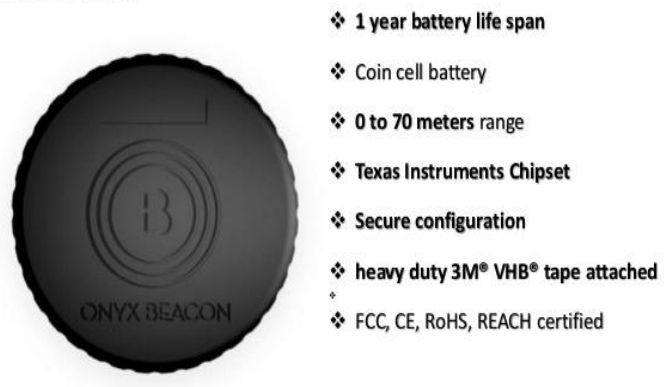

HARDWARE: BEACON ONE

\section{Fig: 1 Onyx Beacon Specifications}

The hardware consists of a microcontroller(8051 family) with a Buetooth LE radio chip and battery.
The major radio chip manufacturers are Texas Instruments and Nordic Semiconductor.

The batteries used are Lithium Ion densed batteries which are coin sized. Sizes available are $240 \mathrm{mAh}$ (CR2032, small size), $620 \mathrm{mAh}$ (CR2450, medium size) and 1,000 mAh (CR2477, large size). Some also use Alkaline AA batteries which are commonly used in TV remotes and in digital cameras. It provides around $2000 \mathrm{mAh}$ power significantly larger than coin cell batteries. Some beacons are externally powered with the help of USB outlets. Though this feature may replace batteries sometimes this does not provide feasibility of the product.

Beacon's firmware which is the programming code deployed has a significant effect on battery life. Below which are the factors that can be controlled by the firmware:

1. Transmit Power (TX power): As Beacon is seen as an emitting source it can emit signals with both having high power and low power. The received signal strength decreases with increase in the distance that is the range. So high power signals consume a significant amount of energy whereas low power signals would consume less energy for the same distance.

2. Advertising Interval: The rate at which a beacon emits signal is known as its advertising signal. The interval ranges between $100 \mathrm{~ms}$ and $500 \mathrm{~ms}$ for a standard beacon. With $100 \mathrm{~ms}$ as the interval the beacon could send signals for every $100 \mathrm{~ms}$ of a second. As a result this would increase the phone's response time significantly but with $500 \mathrm{~ms}$ as the interval the beacon would send signals only twice per second. Though this decreases the response time but improves its battery life. For good experience $500 \mathrm{~ms}$ is more than sufficient.

There are many protocols for configuring hardware. Some beacons like Kontakt, Estimote and RadBeacon configure by using their own proprietary software but other beacons like Minew provide open software through any GATT client enabling it to configure hundred beacons at once.

There are four important chipsets namely: Texas Instruments' TI CC254x, Nordic Semiconductor's nRF51822, Bluegiga's BLE112 / BLE113, and lastly Gimbal's proprietary controller. These occupy $95 \%$ of the market share. 
ISSN(Online) : 2456-8910

International Journal of Innovative Research in Applied Sciences and Engineering (IJIRASE)

Volume 1, Issue 12, DOI: 10.29027/IJIRASE.v1.i12.2018.241-244, June 2018

the request sent by the user. When the bus arrives the bus stops the driver announces its arrival through a microphone that is installed in the bus so that a blind person can easily recognize the bus's arrival at the stop.

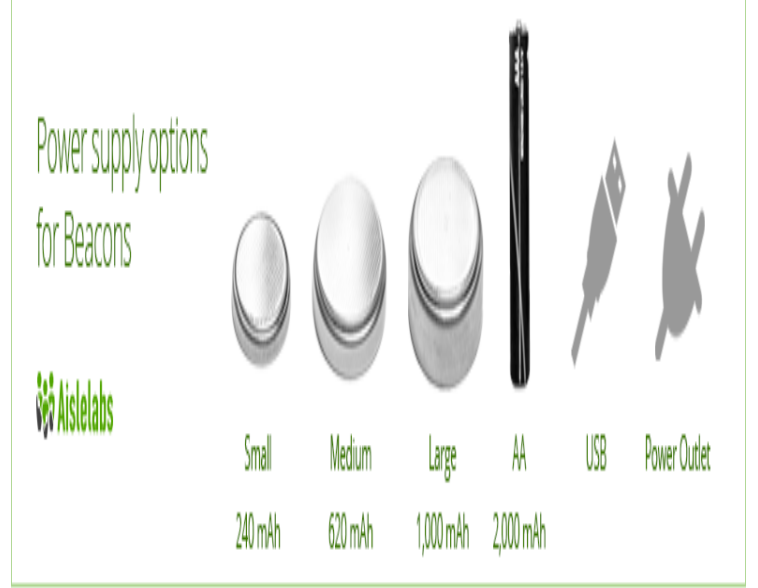

Fig: 2 Various Sizes of A Beacon

\section{III.PROPOSED WORK}

The system design consists of both hardware and software requirements. The hardware unit consist of a bluetooth beacon installed in all the bus stops and buses. The software unit consists of an app(CMSContent Management Systems) installed in smartphones. Google Inc. provides an app called Beacon Tools which enables the user to scan beacons nearby. The beacons may be Eddystone compliant or iBeacons.

The proposed model works as follows:

\section{INITIAL DESIGN}

This system has been designed in such a way to help visually challenged people to board the buses easily. A Bluetooth enabled beacon is installed in the bus stop. The app is installed on the user's smartphone. The beacon is able to recognize the user within five meters with the help of the Bluetooth signal emitted by the user's smartphone. Also the beacon recognizes an approaching bus and it sends the bus's information to the user. But for multiple buses approaching the stop not all the buses may stop at the bus stop. So to overcome this problem a transit web service is established which connects all the buses in the city. Every bus is intended to send its GPS coordinates to the transit web services. These services update the information sent by the bus frequently, manipulates the time of arrival of the bus and sends the necessary information to the user through the app according to

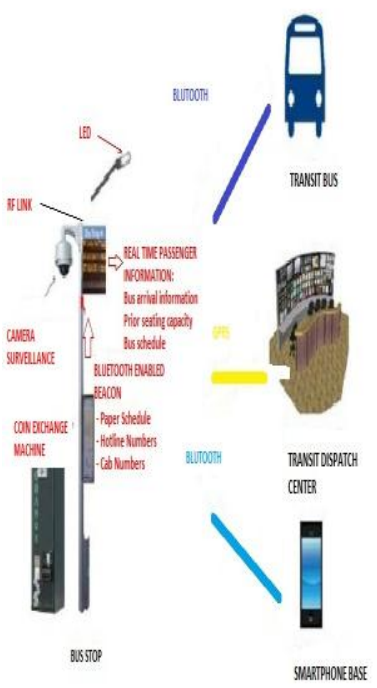

Fig: 3 Initial Design

\section{USER INTERFACE}

The user gets to know all the information regarding the bus timings instantly through the app which is installed in the user's smartphone. This app is based on Advanced Traveller Information Systems(ATIS). The user can check the schedules and can also download the real time transit data via Bluetooth. For visually challenged people a text to speech converter integrated with the app would be a timely help for recognizing approaching buses. Also the app tends to support trip planners and seat availability in the selected bus.

\section{BUS STOP DESIGN}

The beacon is installed in the bus stop and in all the buses. Also real time transit data is displayed on the LED display and even announcements are made exactly the same way in which announcements are made in railway stations and airports. The bus stop recognizes the user with the help of the beacon within five meters. After it recognizes the user a temporal 
ISSN(Online) : 2456-8910

International Journal of Innovative Research in Applied Sciences and Engineering (IJIRASE)

Volume 1, Issue 12, DOI: 10.29027/IJIRASE.v1.i12.2018.241-244, June 2018

connection is established with the user and the beacon. This connection allows the app to transfer all the information that the user wanted. An RF link is also established between the bus stop and the transit workstation.

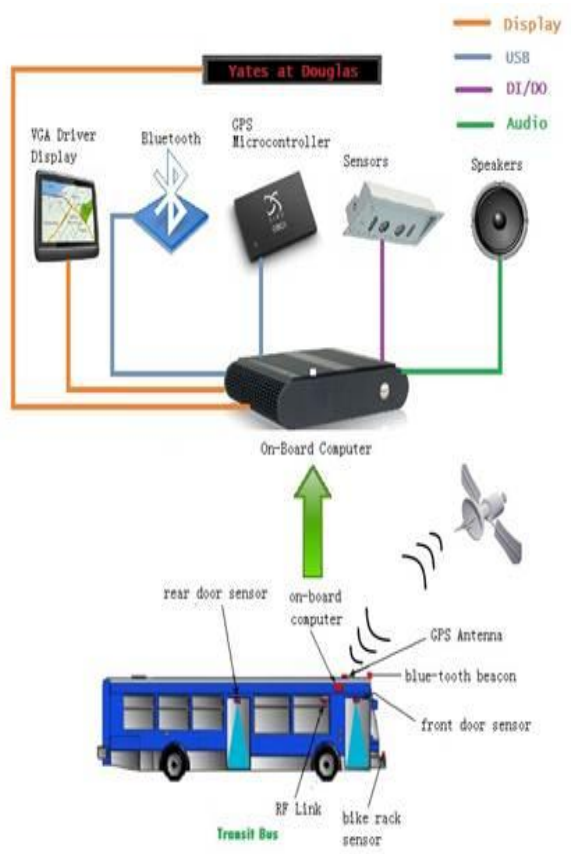

Fig: 4 Bus Stop Design
Technology For Positioning And Tracking In Indoor Place.”, International Journal of Advanced Research in Computer and Communication Engineering Vol. 5, Issue 3, March 2016.

[2] Jingjing Yang, Zhihui Wangand, Xiao Zhang, "An iBeacon based Indoor Positioning Systems for Hospitals." International Journal of Smart Home Vol. 9, No. 7 (2015), pp. 161-168.

[3] https://www.onyxbeacon.com/world-premierelarge-scale-ibeacons-network-guides-visuallyimpaired-people-to-use-the-public-transportationservice/

[4] https://www.onyxbeacon.com/

[5] A. Joseph Ruffa, Amy Stevens, Nicholas Woodward, Torin Zonfrelli, "Assessing iBeacons as an Assistive Tool for Blind People in Denmark.", An Interactive Qualifying Project Denmark Project Center May 2, 2015.

[6] Fard, Hadis Kakanej adi; Chen, Yuanzhu; Son, Kyung Kook, "Indoor positioning of mobile devices with agile iBeacon deployment" 28th Canadian Conference on Electrical and Computer Engineering (CCECE), IEEE, Pages: 275 -279, 2015.

\section{IV.CONCLUSION}

In this paper we have realized a Beacon enabled smart transportation system for the blind. As there is an increase in the number of blind people across the globe this technology would be the need of the hour. This state of art technology helps and guides the blind to travel across the globe without any formalities. Though this technology has few disadvantages, cities like San Francisco has deployed the above mentioned technology in Airports. Also in London underground metro stations have been equipped with this technology and even got positive results from the blind. If possible this technology can be disseminated to different countries so that the blind can lead their life independently.

\section{REFERENCES}

[1] Mr. Sehul A. Thakkar, Mr. Sunil Patel, Mr. Brijesh Kamani "iBeacon: Newly Emerged 